On the other hands, baseline DAS28 for the 20 patients was $4.8 \pm 0.3(2.5-6.8)$ in TCZ group. There were no differences between ADA and TCZ groups. RA patients with an insufficient response to ADA or TCZ showed highly significant improvement of DAS28 after 12 weeks (2.9 \pm 0.3 and $2.2 \pm 0.4$, respectively), and 24 weeks $(2.5 \pm 0.4$ to $2.2 \pm 0.2$, respectively). ADAM-10 highly correlates with CDAI, and fractalkine/CX3CL1. Serum ADAM-10 levels were no remarkable change after treatment with ADA despite decrease of disease activity of RA. On the other hand, serum ADAM-10 levels in patients who were treated with TCZ were significantly diminished following successful treatment and clinical improvement (baseline $408 \pm 88 \mathrm{pg} / \mathrm{ml}$ and 54 weeks $138 \pm 51 \mathrm{pg} / \mathrm{ml}, \mathrm{p}<0.05$ ). Univariate logistic regression analysis, baseline of DAS28 (ESR), baseline of CDAI, and ADAM-10 were selected as significant variables for improvement of DAS28 (ESR) at 24 weeks. Multiple regression analysis showed that ADAM-10 was only identified as independent predictive variable for improvement of DAS28 (ESR) at 24 weeks. ADAM-10 baseline in TCZ responder was significantly higher than TCZ nonresponders at 24 weeks $(620 \pm 134 \mathrm{pg} / \mathrm{ml}$ and $109 \pm 25 \mathrm{pg} / \mathrm{ml}$, respectively, $\mathrm{p}<0.05)$.

Conclusions: This study indicates that ADAM-10 is correlated with RA disease activity, and is higher in TCZ responders. These results suggest that ADAM-10 may be a predictor of treatment effectiveness for RA with TCZ.

Disclosure of Interest: None declared

DOI: 10.1136/annrheumdis-2017-eular.4594

\section{THU0065 CYTOKINES AND LIPOCALIN-2 IN PREGNANT WOMEN WITH RHEUMATOID ARTHRITIS AND SYSTEMIC LUPUS ERYTHEMATOSUS}

T.T. Pedersen ${ }^{1}$, M.H. Fenstad ${ }^{2}$, T.S. Moksnes ${ }^{1}$, M. Wallenius ${ }^{1}$, T.H. Flo ${ }^{3}$, M. Haug ${ }^{3} \cdot{ }^{1}$ Norwegian National Advisory Unit on Pregnancy and Rheumatic diseases; ${ }^{2}$ Dep. of Immunology and Transfusion Medicine, St. Olavs Hospital; ${ }^{3}$ Centre of Molecular Inflammation Research and Dep. of Cancer Research and Molecular Medicine, NTNU, Trondheim, Norway

Background: Rheumatoid arthritis (RA), and especially seronegative RA, is often ameliorated by pregnancy, while systemic lupus erythematosus (SLE) is prone to flare and associated with pregnancy complications. Cytokines and chemokines are of great importance for immune processes during pregnancy. The inflammatory marker Lipocalin-2 (LCN2) has become increasingly relevant as a potential clinical biomarker of rheumatic diseases (1). LCN2 is produced in the maternal-fetal interface during normal pregnancies and correlates with the presence and severity of preeclampsia (2).

Objectives: To obtain a better understanding of immune regulation and the disparate immune responses in pregnant women with RA and SLE. In this pilot study, we analyzed levels of multiple cytokines, chemokines and LCN2 in women with seropositive RA, seronegative RA, SLE and healthy controls during pregnancy and postpartum

Methods: The Norwegian National Advisory Unit on Pregnancy and Rheumatic Diseases collect serum samples in a biobank from women with inflammatory rheumatic diseases before pregnancy, during pregnancy week 10-12, week 23-25, week 30-32 and 6 weeks, 6 months and 12 months postpartum. Control serum samples were collected from healthy pregnant women at matching timepoints. We analyzed serum cytokine and chemokine levels using a multiplex assay. A sandwich ELISA was used to measure LCN2. In this pilot study we included pregnant women with SLE $(n=4)$, seropositive RA $(n=4)$, seronegative RA $(n=2)$ and healthy pregnant controls $(n=4)$. The total cohort consists so far of 18 pregnant women with SLE and 23 pregnant women with RA.

Results: We observed lower LCN2 levels during pregnancy in SLE patients, compared to controls and RA patients. LCN2 levels in seropositive RA patients and controls were found to be comparable during pregnancy, whereas pregnant women with seronegative RA showed higher LCN2 levels. Levels of IFN $\gamma$, IL-6 and IP-10 were higher in SLE than in RA patients during the course of pregnancy. IL-17 was slightly higher only in seropositive RA patients compared to controls. TNF $\alpha$ was slightly higher in both SLE and RA patients compared to controls, levels of anti-inflammatory IL-10 were very low or undetectable in all groups.

\begin{tabular}{lcccc}
\hline 2. Trimester & Controls & SLE & Seropos RA & Seroneg RA \\
\hline LCN2 $(\mathrm{ng} / \mathrm{ml})$ & $285,5( \pm 74,7)$ & $126,5( \pm 44,6)$ & $232,6( \pm 67,4)$ & $330,2( \pm 3,2)$ \\
IFN $\gamma(\mathrm{pg} / \mathrm{ml})$ & $15,0( \pm 22,4)$ & $40,4( \pm 65,1)$ & $6,9( \pm 5,2)$ & $26,1( \pm 9,9)$ \\
IL-6 $(\mathrm{pg} / \mathrm{ml})$ & $3,4( \pm 1,9)$ & $5,9( \pm 2,0)$ & $3,8( \pm 1,5)$ & $5,3( \pm 1,0)$ \\
IP-10 $(\mathrm{pg} / \mathrm{ml})$ & $60,3( \pm 14,5)$ & $138,3( \pm 92,1)$ & $61,7( \pm 8,9)$ & $64,9( \pm 7,3)$ \\
IL-17 $(\mathrm{pg} / \mathrm{ml})$ & $1,3( \pm 1,3)$ & $2,3( \pm 2,2)$ & $4,8( \pm 9,0)$ & $0,6( \pm 0,2)$ \\
TNF $\alpha(\mathrm{pg} / \mathrm{ml})$ & $2,2( \pm 2,5)$ & $4,2( \pm 5,4)$ & $3,3( \pm 3,5)$ & $3,2( \pm 0,7)$ \\
\hline
\end{tabular}

Conclusions: We found interesting differences in cytokine, chemokine and LCN2 levels during pregnancy in women with SLE, seropositive RA and seronegative RA. The results need confirmation in the total cohort and will be further explored for a better understanding of the disparate immune modulation of RA and SLE during pregnancy.

References:

[1] Abella V et al. Biomarkers. 2015;20(8):565-71.

[2] Kim SM et al. Reproductive sciences. 2013;20(9):1083-9.

Acknowledgements: This study is supported by grants from St. Olavs Hospital and Norsk Revmatikerforbund.
Disclosure of Interest: None declared

DOI: 10.1136/annrheumdis-2017-eular.1916

\section{THU0066 OSTEOCLAST DIFFERENTIATION GENE EXPRESSION PROFILING REVEALS CCL4 MEDIATES RANKL-INDUCED OSTEOCLAST MIGRATION}

W. Xuan ${ }^{1}$, X. Feng ${ }^{2}$, Y. Shi ${ }^{1}$, F. Wang ${ }^{3}$, M. Zhang ${ }^{1}$, W. Tan ${ }^{1} .{ }^{1}$ Rheumatology; ${ }^{2}$ Traditional Chinese Medicine; ${ }^{3}$ Department of Cardiology, the First Affiliated Hospital of Nanjing Medical University, China, nanjing, China

Background: The migration of osteoclast from circulation and bone marrow into bone surface has suggested as a novel therapeutic point for bone erosion in RA. Objectives: We explored the mechanisms involved in osteoclast migration. Methods: Gene expression profiling was identified by microarray analysis and validated by Real-time PCR during differentiation of bone marrow-derived macrophages (BMMs) into osteoclast (OCs). RANKL induced osteoclast precursor cell line RAW264.7 migration and invasion in the presence and absence of anti-CCL4 antibody was measured in vitro. Intracellular signaling pathway was assessed by Western blotting. Osteoclast formation was identified by TRAP staining. Results: A panel of 11 chemokines signal was significant increase in osteoclastic differentiation of BMMs by Microarray. High expression of CCL4 was validated in primary BMMs and RAW264.7 cell line during differentiated into OCs. RANKL induced osteoclast precursor cell migration and invasion was decreased upon addition of anti-CCL4 antibody. OCs formation and OCs related genes expression were not affected by CCL4 inhibition. Neutralization of CCL4 promoted the PI13K phosphorylation at 45 to 60 min after RANKL stimulation in RAW264.7.

Conclusions: CCL4 regulates RANKL-induced OCs migration, suggesting that CCL4 inhibition could be bone protective in RA

\section{References:}

[1] Boyce BF: Advances in the regulation of osteoclasts and osteoclast functions. Journal of dental research 2013, 92(10):860-867.

[2] Crotti TN, Dharmapatni AA, Alias E, Haynes DR: Osteoimmunology: Major and Costimulatory Pathway Expression Associated with Chronic Inflammatory Induced Bone Loss. Journal of immunology research 2015, 2015:281287.

[3] Boyle WJ, Simonet WS, Lacey DL: Osteoclast differentiation and activation. Nature 2003, 423(6937):337-342.

Acknowledgements: Support for this work was obtained from the National Natural Science Foundation of China (NSFC): 30701129 (WT), 30901332 (FW), 81172845 (WT), 81273294 (MZ), National Natural Science Foundation of Jiangsu province: BK2011851 (WT), BK2012875 (MZ), the special project of clinical medicine from Jiangsu province: BL2013034 (MZ), A Project Funded by the Priority Academic Program Development of Jiangsu Higher Education Institutions (PAPD), and scholarship from Asia Pacific League of Associations for Rheumatology (APLAR) and International League of Associations for Rheumatology (ILAR) (WT).

Disclosure of Interest: None declared

DOI: 10.1136/annrheumdis-2017-eular.4255

\section{THU0067 NLRP3 INFLAMMASOME ACTIVITY IN MONOCYTES IS REGULATED BY 12/15-LIPOXYGENASE}

Y. Kusche, K. Barczyk-Kahlert, J. Roth. Institut Der Immunologie, Münster, Germany

Background: Activation of the NLRP3 inflammasome is a major inflammatory pathway in monocytes in response to various exogenous and endogenous stimuli. However, negative regulation of inflammasome activity is not well understood. Glucocorticoids (GC) are drugs of choice for the treatment of many inflammatory diseases. Recently, we could show that treatment of monocytes with GC leads to re-programming towards a specific population involved in resolution of inflammation. Gene analysis has shown up-regulated expression of 12/15-lipoxygenase (12/15-LOX) in GC-and LPS/GC-treated monocytes. 12/15LOX reacts with polyunsaturated-fatty-acids to generate anti-inflammatory lipidmediators, which contribute to resolution of inflammation.

Objectives: The aim of our study was to determine the contribution of 12/15-LOX on the inflammatory response on murine monocytes.

Methods: Bone marrow-derived monocytes were isolated from wild-type (wt) C57BL/6 and 12/15-LOX $\%$ mice and stimulated with GC and/or LPS as well as various inhibitors or stimulants. Gene expression was analyzed using qRT-PCR. Protein expression was examined by Western-Blot, Flow-Cytometry and ELISA. T-cell response was analyzed by co-culture of stimulated monocytes with allogenic T-cells.

Results: $12 / 15-\mathrm{LOX}^{-/-}$monocytes showed slightly higher secretion of IL-1 $\beta$ as compared to wt cells after LPS stimulation. The differences between wt and $12 / 15-$ LOX $^{-/}$were much more pronounced when monocytes were additionally exposed to ATP. LPS treatment markedly enhanced expression of pro-IL-1 $\beta$ in $12 / 15-$ LOX $^{-/-}$monocytes. No differences could be observed between wt and $12 / 15-$ LOX $^{-/}$monocytes in secretion of other proinflammatory mediators as well as the expression of inflammasome components. However, expression of cleaved caspase-11 was up-regulated in 12/15-LOX ${ }^{-/}$monocytes exposed to LPS. Additionally, inhibition of caspase-11, caspase-1 and 5-LOX significantly reduced the high secretion of IL-1 $\beta$ in $12 / 15$ LOX $^{-/-}$monocytes. Interestingly, 12/15-LOX ${ }^{-/-}$ rather than wt monocytes stimulated with LPS led to enhanced T-cell proliferation. 